\title{
PROJECTIVITY OF THE WHITEHEAD PRODUCT IN SPHERES
}

\author{
WEN-HSIUNG LIN
}

(Communicated by Frederick R. Cohen)

Abstract. The Whitehead square $\left[l_{2^{i}-1}, l_{2^{i}-1}\right] \in \pi_{2^{i+1}-3}\left(S^{2^{i}-1}\right)$ is shown to be projective for $i \leq 10$.

\section{INTRODUCTION}

A homotopy element in $\pi_{n}(X)$ is said to be projective if it can be represented by a map $f: S^{n} \rightarrow X$ that factors through the real projective space $P^{n}$, that is, the diagram

$$
\begin{array}{r}
S^{n} \stackrel{f}{\longrightarrow} X \\
\quad \underset{P^{n}}{\nearrow_{f}} \bar{f}
\end{array}
$$

commutes for some $\bar{f}$, where $\sigma$ is the double covering map. The notion of projective homotopy elements was introduced by J. H. C. Whitehead [21]. Some research has been done on the projective homotopy theory and its applications in the last two decades. For this we refer to [17, §1].

Here we will be interested in the problem of the projectivity of the Whitehead products $\left[l_{n}, l_{n}\right] \in \pi_{2 n-1}\left(S^{n}\right)$. The status of the problem so far is the following.

D. Randall proves in [18] that $\left[l_{n}, l_{n}\right]$ is not projective with $n$ nor $n+1$ a power of two. Milgram and Zvengrowski show [16] that $\left[l_{2^{m}}, l_{2^{m}}\right]$, for $m \geq 1$, is projective iff $m=1,2,3$. It remains to consider $\left[l_{2^{i}-1}, l_{2^{i}-1}\right]$. It is well known that $\left[l_{2^{i}-1}, l_{2^{i}-1}\right]=0$ for $1 \leq i \leq 3$; so they are projective trivially. Adams Hopf invariant one theorem [1] implies $\left[l_{2^{i}-1}, l_{2^{i}-1}\right] \neq 0$ for $i \geq 4$. The problem of the projectivity of the family $\left\{\left[l_{2^{i}-1}, l_{2^{i}-1}\right] \mid i \geq 4\right\}$ is related to another well-known problem on the same family; namely, the so-called strong Kervaire invariant problem which asks whether $\left[l_{2^{i}-1}, l_{2^{i}-1}\right](i \geq 4)$ can be halved. It is easy to see that if $\left[l_{2^{i}-1}, l_{2^{i}-1}\right]$ can be halved, then it is projective. Toda shows in [20] that $\left[l_{15}, l_{15}\right]=2 \sigma^{2}$, and Mahowald and Tangora prove in $[15]$ that $\left[l_{31}, l_{31}\right]=2 \theta_{4}$; so $\left[l_{15}, l_{15}\right]$ and $\left[l_{31}, l_{31}\right]$ are projective. The conjecture for $i \geq 6$ is presently unknown.

Received by the editors May 1, 1989.

1980 Mathematics Subject Classification (1985 Revision). Primary 55P40, 55Q15, 55T15.

The author was partially supported by the National Science Council of the Republic of China. 
Our purpose here is to show that the answer to the projectivity problem about $\left[l_{2^{i}-1}, l_{2^{i}-1}\right]$ is positive in a few dimensions higher. The result is the following.

Theorem 1.1. The Whitehead product $\left[l_{2^{i}-1}, l_{2^{i}-1}\right] \in \pi_{2^{i+1}-3}\left(S^{2^{i}-1}\right)$ is projective for $i \leq 10$.

We conjecture this result is true for all $i$.

\section{Proof}

For $1 \leq k<l$ let $P_{k}^{l}$ denote the stunted real projective space $P^{l} / P^{k-1}$. We will show the following.

Proposition 2.1. Let $m=2^{i}-1, \bar{m}=2^{i+1}-2^{i-3}-1 \quad(i \geq 3)$. Then for $i \leq 10$ there is a map $g: P_{\bar{m}}^{2 m-1} \rightarrow S^{m}$ such that the composite

$$
S^{2 m-1} \stackrel{\bar{\sigma}}{\longrightarrow} P_{\bar{m}}^{2 m-1} \stackrel{g}{\longrightarrow} S^{m}
$$

represents the Whitehead product $\left[l_{m}, l_{m}\right]$.

Here $\bar{\sigma}$ is the composite $S^{2 m-1} \stackrel{\sigma}{\longrightarrow} P^{2 m-1} \stackrel{q}{\longrightarrow} P_{\bar{m}}^{2 m-1}$ with $q$ the collapsing map. Note that the mapping cone of $\bar{\sigma}$ is $P_{\bar{m}}^{2 m}$. Then, according to [4, Theorem 3.1], (2.1) is equivalent to

Proposition 2.1" . Let $m, \bar{m}$ be as in (2.1). For $i \leq 10$, there is a stable map $\bar{g}: \sum P_{\bar{m}}^{2 m} \rightarrow S^{m+1}$ such that in $X=S^{m+1} \cup_{\bar{g}} C \sum P_{\bar{m}}^{2 m}$ the Steenrod operation

$$
S q^{m+1}=S q^{2^{i}}: H^{m+1}(X)=\mathbb{Z}_{2} \rightarrow H^{2 m+2}(X)=\mathbb{Z}_{2}
$$

is nonzero.

Here, and also for the rest of the paper, all cohomology or homology groups have $\mathbb{Z}_{2}$ coefficients.

Let $B(k)$ denote the $k$ th 2-primary Brown-Gitler spectrum [7]. Recall that $H^{*}(B(k))=A / A\left\{\chi\left(S q^{j}\right) \mid j>k\right\}$ where $A$ is the mod 2 Steenrod algebra and $\chi: A \rightarrow A$ is the canonical antiautomorphism. Then $\left\{\chi\left(S q^{I}\right) \mid I=\right.$ $\left(i_{1}, \ldots, i_{k}\right)$ admissible and $\left.i_{1} \leq k\right\}$ is a $\mathbb{Z}_{2}$-base for $H^{*}(B(k))$. In particular $H^{2^{j+1}-1}\left(B\left(2^{j}\right)\right)=\mathbb{Z}_{2}$ is generated by $\chi\left(S q^{2^{j}} S q^{2^{j-1}} \cdots S q^{2} S q^{1}\right)$ and $H^{k}\left(B\left(2^{j}\right)\right)$ $=0$ for $k>2^{j+1}-1$.

Consider the double loop space $\Omega^{2} S^{9}$. There is a 2-primary stable decomposition

$$
\Omega^{2} S^{9} \simeq \bigvee_{k=1}^{\infty} \sum^{7 \cdot k} B\left(\left[\frac{k}{2}\right]\right)
$$

This is due to Brown-Peterson, Mahowald, and Snaith $[8,14,19]$. Let $S^{0}$ denote the sphere spectrum in stable dimension zero. Mahowald also shows the following in [14]. 
Proposition 2.2 [14]. There is a stable map $f: \Omega^{2} S^{9} \rightarrow S^{0}$ such that if $f_{i}=$ $f \mid \sum^{7 \cdot 2^{i-3}} B\left(2^{i-4}\right)$ then in the mapping cone $X_{i}$ of $F_{i}: \sum^{7 \cdot 2^{i-3}} B\left(2^{i-4}\right) \rightarrow S^{0}$ the Steenrod operation

$$
S q^{2^{i}}: H^{0}\left(X_{i}\right)=\mathbb{Z}_{2} \rightarrow H^{2^{i}}\left(X_{i}\right)=\mathbb{Z}_{2}
$$

is nonzero for all $i \geq 3\left(B\left(2^{i-4}\right)=B(0)\right.$ if $\left.i=3\right)$.

We will show the following.

Proposition 2.3. Let $m=2^{i}-1, \bar{m}=2^{i+1}-2^{i-3}-1$. For $3 \leq i \leq 10$ there is a stable map $\phi: \sum^{-\bar{m}} P_{\bar{m}}^{2 m} \rightarrow B\left(2^{i-4}\right) \quad\left(B\left(2^{i-4}\right)=B(0)\right.$ if $\left.i=3\right)$ such that $\phi^{*}: H^{0}\left(B\left(2^{i-4}\right)\right)=\mathbb{Z}_{2} \rightarrow H^{0}\left(\sum^{-\bar{m}} P_{\bar{m}}^{2 m}\right)=\mathbb{Z}_{2}$ is nonzero.

This implies $\phi^{*}: H^{2^{i-3}-1}\left(B\left(2^{i-4}\right)\right)=\mathbb{Z}_{2} \rightarrow H^{2^{i-3}-1}\left(\sum^{-\bar{m}} P_{\bar{m}}^{2 m}\right)=\mathbb{Z}_{2}$ is nonzero since $\chi\left(S q^{2^{i-4}} S q^{2^{i-5}} \cdots S q^{2} S q^{1}\right)=S q^{2^{i-3}-1}: H^{0}\left(\sum^{-m} P_{\bar{m}}^{2 m}\right)=\mathbb{Z}_{2} \rightarrow$ $H^{2^{i-3}-1}\left(\sum^{-\bar{m}} P_{\bar{m}}^{2 m}\right)=\mathbb{Z}_{2}$ is nonzero [11]. Proposition $2.1^{\prime}$, and therefore Theorem 1.1, follows from this and (2.2).

Note that the Spanier-Whitehead dual of

$$
\sum^{-2^{i+1}+1} P_{\bar{m}}^{2 m}=\sum^{-2^{i+1}+1} P_{2^{i+1}-2^{i-3}-1}^{2^{i+1}-2}
$$

is

$$
\sum^{2^{i+1}} P_{-2^{i+1}+1}^{-2^{i+1}+2^{i-3}}
$$

$[2,3]$. Then $(2.3)$ is equivalent to

Proposition 2.3" . Let

$$
X=\sum^{2^{i+1}} P_{-2^{i+1}+1}^{-2^{i+1}+2^{i-3}} .
$$

For $3 \leq i \leq 10$ there is a stable map $\Phi: S^{2^{i-3}} \rightarrow B\left(2^{i-4}\right) \wedge X$ such that $\bar{\phi}^{*}(1 \otimes \nu) \neq 0$ where $\nu$ is the top nonzero class of $H^{*}(X)$.

To prove $\left(2.3^{\prime}\right)$ we use the method in [6].

Let $Y$ be a spectrum. Consider the $\bmod 2$ Adams spectral sequence $\left\{E_{r}^{*, *}(k)\right\}$ for computing $\pi_{*}^{S}(B(k) \wedge Y)$. An $E_{1}$-term may be chosen as follows.

Let $\Lambda$ be the lambda algebra of Kan et al. [5]. $\Lambda$ is a bigraded algebra over $\mathbb{Z}_{2}$ generated by $\lambda_{i} \in \Lambda_{i}^{1} \quad(i \geq 0)$ and has the set of admissible monomials $\left\{\lambda_{i_{1}} \lambda_{i_{2}} \cdots \lambda_{i_{s}} \mid 2 i_{j} \geq i_{j+1}\right\}$ as a $\mathbb{Z}_{2}$-base. Let $\Lambda(k) \subset \Lambda$ be the $\mathbb{Z}_{2}$-submodule generated by $\left\{\lambda_{I} \mid I=\left(i_{1}, \ldots, i_{s}\right)\right.$ admissible and $\left.i_{s} \geq k\right\}$. Then

$$
E_{1}^{s, t}(k)=\sum_{p} \Lambda_{t-s-p}^{s}(k) \otimes H_{p}(Y)
$$

with differential

$$
d_{1}\left(\lambda_{I} \otimes u\right)=\sum_{j \geq 0} \lambda_{j} \lambda_{I} \otimes u S q^{j+1},
$$

where the right action of the Steenrod algebra $A$ on $H_{*}(Y)$ is obtained by transposing the left action of $A$ on $H^{*}(Y)$. 
Lemma 2.4 [6]. If $H_{*}(Y)$ is an unstable module over $A$, that is, $u S q^{j}=0$ for $|u|<2 j$, then $d_{1}: E_{1}^{s, t}(k) \rightarrow E_{1}^{s+1, t}(k)$ is zero for $t-s \leq 2 k+1$.

Given an integer $n \geq 0$. Impose a condition " $C_{n}$ " on $Y$ as follows. For $n=0, Y$ satisfies " $C_{0}$ " if $H_{l}(Y)=0$ for $l<0$, and $H_{*}(Y)$ is an unstable $A$ module. For $n>0, Y$ satisfies " $C_{n}$ " if there are a spectrum $W$ that satisfies " $C_{n-1}$ " and a map $f: Y \rightarrow W$ such that $f_{*}: H_{*}(Y) \rightarrow H_{*}(W)$ is monomorphic and for any $u \in H_{l}(Y)$ there exists $\nu \in H_{2 l}(W)$ with $\nu S q^{l}=f_{*}(u)$. We also allow $n=\infty ; Y$ satisfies " $C_{\infty}$ " if is satisfies " $C_{n}$ " for all integers $n \geq 0$.

\section{Examples.}

$Y$ is said to have spacelike homology if it admits a map to the suspension spectrum of a CW complex, $f: Y \rightarrow W$ such that $f_{*}: H_{*}(Y) \rightarrow H_{*}(W)$ is a monomorphism. It is proved in [6] that if $Y$ has spacelike homology then $Y$ satisfies " $C_{\infty}$ ". In particular, all suspension spectra of $C W$ complexes satisfy " $C_{\infty}$ ".

(2.5.2) For $i \geq 3$, the spectra

$$
\sum^{2^{i+1}} P_{-2^{i+1}+1}^{-2^{i+1}+l} \text { for } 1 \leq l \leq 2^{i-3}
$$

satisfy " $C_{4}$ ". To see this we note that

$$
H^{*}\left(\sum^{2^{i+1}} P_{-2^{i+1}+1}^{-2^{i+1}+l}\right), \quad \text { for } 1 \leq l \leq 2^{i+1}
$$

is an unstable $A$-module, that

$$
\sum^{2^{i+1}} P_{-2^{i+1}+1}^{-2^{i+1}+l} \subset \sum^{2^{i+1}} P_{-2^{i+1}+1}^{0}
$$

and that, in $H^{*}\left(\sum^{2^{i+1}} P_{-2^{i+1}+1}^{0}\right), S q^{8|u|} S q^{4|u|} S q^{2|u|} S q^{|u|} u \neq 0$ for nonzero $u \in H^{|u|}\left(\sum^{2^{i+1}} P_{-2^{i+1}+1}^{0}\right)$ with $1 \leq|u| \leq 2^{i-3}$.

Remark 2.6. Davis and Mahowald prove in [12] that

$$
X=\sum^{2^{i+1}} P_{-2^{i+1}+1}^{-i^{i+1}+2^{i-3}}
$$

and $P_{1}^{2^{i-3}}$ (for $i \geq 3$ ) have the same stable homotopy type if and only if $i \leq 6$. Thus, for $i \leq 6, X$ has spacelike homology. It is not difficult to show that if $X$ has spacelike homology then $X$ has the stable homotopy type of $P_{1}^{2^{i-3}}$. Thus, for $i \geq 7, X$ has no spacelike homology.

Proposition 2.7. For $0 \leq n \leq \infty$, if $Y$ satisfies " $C_{n}$ " then $d_{r}: E_{r}^{s, t}(k) \rightarrow$ $E_{r}^{s+r, t+r-1}(k)$ is zero for $t-s \leq 2 k+1$ and $r \leq n+1$.

The proof is given later.

Applying (2.7) to $Y=S^{0}$, we see, by (2.5.1), $E_{\infty}^{s, t}(k)=\Lambda_{t-s}^{s}(k)$ for $t-s \leq$ $2 k$. Brown and Peterson prove in [9] the following sharper result. 
Proposition 2.8 [9]. As a group, $\pi_{t-s}^{s}(B(k)) \simeq \Lambda_{t-s}^{s}(k)$ for $t-s<2 k$.

Now we deduce from (2.7) and (2.8) Proposition 2.3'.

Proof of Proposition $2.3^{\prime}$. We apply the spectral sequence to

$$
Y=X=\sum^{2^{i+1}} P_{-2^{i+1}+1}^{-2^{i+1}+2^{i-3}}
$$

in $\left(2.3^{\prime}\right)$. Let $u$ be the nonzero class of $H_{2^{i-3}}(Y)=\mathbb{Z}_{2}$. Then $1 \otimes u \in$ $E_{1}^{0,2^{i-3}}\left(2^{i-4}\right)$. To prove $\left(2.3^{\prime}\right)$ is to show

$$
1 \otimes u \text { persists to } E_{\infty}^{0,2^{i-3}}\left(2^{i-4}\right) \quad \text { for } \quad i \leq 10 .
$$

By (2.5.2) and (2.7), $d_{r}(1 \otimes u)=0$ for $r \leq 5$. We have to show $d_{r}(1 \otimes u)=0$ for $r \geq 6$.

Recall $\Lambda_{2^{i-3}-1-p}^{r}\left(2^{i-4}\right)$ has $\left\{\lambda_{I} \mid I=\left(i_{1}, \ldots, i_{r}\right)\right.$ admissible, $i_{r} \geq 2^{i-4}$, and $\left.|I|=2^{i-3}-1-p\right\}$ as a $\mathbb{Z}_{2}$-base. One easily sees, then, that $\Lambda_{2^{i-3}-1-p}^{r}\left(2^{i-4}\right)=0$ for $r \geq 6,3 \leq i \leq 9$, and $1 \leq p \leq 2^{i-3}$. Thus $d_{r}(1 \otimes u)=0$ for $r \geq 6$ since

$$
\begin{aligned}
d_{r}(1 \otimes u) & \in E_{r}^{r, 2^{i-3}+r-1}\left(2^{i-4}\right) \subset E_{1}^{r, 2^{i-3}+r-1}\left(2^{i-4}\right) \\
& =\sum_{p} \Lambda_{2^{i-3}-1-p}^{r}\left(2^{i-4}\right) \otimes H_{p}(Y)=0 .
\end{aligned}
$$

This proves $(*)$ for $i \leq 9$.

For $i=10, r \geq 6,1 \leq p \leq 2^{i-3}=2^{7}$, the $\mathbb{Z}_{2}$-module $\Lambda_{2^{i-3}-1-p}^{r}\left(2^{i-4}\right)=$ $\Lambda_{2^{7}-1-p}^{r}\left(2^{6}\right)$ is zero except for $r=6, p=1$ in which case $\Lambda_{2^{7}-2}^{6}\left(2^{6}\right)=\mathbb{Z}_{2}$ and is generated by $\lambda_{2} \lambda_{4} \lambda_{8} \lambda_{16} \lambda_{32} \lambda_{64}$. So to prove (*) for $i=10$ it suffices to show

$$
d_{6}(1 \otimes u)=0 \neq \lambda_{2} \lambda_{4} \lambda_{8} \lambda_{16} \lambda_{32} \lambda_{64} \otimes u_{1},
$$

where $u_{1} \neq 0 \in H_{1}(Y)=\mathbb{Z}_{2}$.

Consider

$$
X^{\prime}=\sum^{2^{11}} P_{-2^{11}+2}^{-2^{11}+2^{7}}=X / S^{1}=\sum^{2^{11}} P_{-2^{11}+1}^{-2^{11}+2^{7}} / S^{1} .
$$

Note that the Spanier-Whitehead dual of $X^{\prime}(\operatorname{resp} . X)$ is

$$
\left.\sum^{-2^{11}+1} P_{2^{11}-2^{71}-1}^{2^{11}-3} \text { (resp. } \sum^{-2^{11}+1} P_{2^{11}-7-1}^{2^{11}-2}\right) \text {. }
$$

Also note that $X^{\prime}$ satisfies " $C_{4}$ ". We apply (2.7) to $X^{\prime}$. The analysis above shows that $1 \otimes u^{\prime} \in E_{1}^{0,2^{7}}\left(2^{6}\right)$ persists to $E_{\infty}$ where $u^{\prime} \neq 0 \in H_{2^{7}}\left(X^{\prime}\right)=\mathbb{Z}_{2}$. This implies there is a stable map

$$
\sum^{-2^{11}+2^{7}+1} P_{2^{11}-2^{7}-1}^{2^{11}-3} \stackrel{h}{\longrightarrow} B\left(2^{6}\right)
$$

that is nonzero in $H_{0}()$. Let $\tau: S^{2^{7}-2} \rightarrow \sum^{-2^{11}+2^{7}+1} P_{2^{11}-2^{11}-1}^{2^{11}}$ be the attaching map for $\sum^{-2^{11}+2^{7}+1} P_{2^{11}-2^{7}-1}^{2^{11}-2}$. Then $d_{6}(1 \otimes u)=\lambda_{2} \lambda_{4} \lambda_{8} \lambda_{16} \lambda_{32} \lambda_{64} \otimes u_{1}$ if and 
only if the composite

$$
S^{2^{7}-2} \stackrel{\tau}{\longrightarrow} \sum^{-2^{11}+2^{7}+1} P_{2^{11}-2^{7}-1}^{2^{11}-3} \stackrel{h}{\longrightarrow} B\left(2^{6}\right)
$$

is detected by $\lambda_{2} \lambda_{4} \lambda_{8} \lambda_{16} \lambda_{32} \lambda_{64}$.

Consider the mapping cone $Z=\left(S^{2^{7}-2} \vee S^{2^{7}-1}\right) \cup e^{2^{7}}$ of $S^{2^{7}-1} \stackrel{(\eta, 2 l)}{\longrightarrow}$ $S^{2^{7}-2} \vee S^{2^{7}-1}$. The map $\tau$ is the restriction of the attaching map

$$
\bar{\tau}: Z \rightarrow \sum^{-2^{11}+2^{7}+1} P_{2^{11}-2^{71}-1}^{2^{11}} \text { for } \sum^{-2^{11}+2^{7}+1} P_{2^{11}-2^{71}-1} .
$$

If $h \tau$ is detected by $\lambda_{2} \lambda_{4} \lambda_{8} \lambda_{16} \lambda_{32} \lambda_{64}$ then $\left[\left.h \bar{\tau}\right|_{S^{2^{7}-1}}\right] \in \pi_{2^{7}-1}\left(B\left(2^{6}\right)\right)$ is an element with $2\left[\left.h \bar{\tau}\right|_{S^{2}{ }^{7}-1}\right]$ detected by $\lambda_{1} \lambda_{2} \lambda_{4} \lambda_{8} \lambda_{16} \lambda_{32} \lambda_{64} \neq 0$ since $\lambda_{1}$ detects $\eta$. In particular, $2\left[\left.h \bar{\tau}\right|_{S^{2^{7}-1}}\right] \neq 0$. But, by Proposition 2.8 , all elements of $\pi_{2^{7}-1}\left(B\left(2^{6}\right)\right)$ have order at most 2 . Thus $h \tau$ cannot be detected by $\lambda_{2} \lambda_{4} \lambda_{8} \lambda_{16} \times$ $\lambda_{32} \lambda_{64}$ that is, $d_{6}(1 \otimes u)=0$. This proves $(*)$ for $i=10$. This completes the proof of Proposition (2.3'). Q.E.D.

The method of the proof of Proposition 2.7 is the one given in [6] where only spectra having space-like homology are considered. We recall the proof.

There are cofibrations $[10,14]$

$$
B(l-1) \wedge Y \stackrel{\beta}{\longrightarrow} B(l) \wedge Y \stackrel{\alpha}{\longrightarrow} \sum^{l} B\left(\left[\frac{l}{2}\right]\right) \wedge Y
$$

natural in $Y . \beta$ and $\alpha$ induce $\beta_{*}: E_{r}^{s, t}(l-1) \rightarrow E_{r}^{s, t}(l)$ and $\alpha_{*}: E_{r}^{s, t}(l) \rightarrow$ $E_{r}^{s, t-l}([l / 2])$. They have the following properties.

1. $\beta_{*}: E_{1}^{s, t}(l-1) \rightarrow E_{1}^{s, t}(l)$ is given by

$$
\beta_{*}\left(\lambda_{I} \otimes u\right)=\lambda_{I} \otimes u
$$

2. (i) For $t-s<2 l, \alpha_{*}: E_{1}^{s, t}(l) \rightarrow E_{1}^{s, t-l}([l / 2])$ is zero and any $\lambda_{I} \otimes u \in$ $E_{1}^{s, t}(l)$ pulls back uniquely to $E_{1}^{s, t}(l-1)$ (as described in 1$)$.

(ii) If $\left|\lambda_{2 I} \otimes u\right|=2 l$ and $u \in H_{2 p}(Y)$ then

$$
\alpha_{*}\left(\lambda_{2 I} \otimes u\right)=\lambda_{I} \otimes u S q^{p}
$$

Proposition 2.7 is proved by induction on $n$ and $k$. The result is obvious for $k=0$. It is also true for $n=0$ by Lemma 2.4. Suppose $n>0, k>0$, and suppose the result is true for either $k^{\prime}<k$ or $n^{\prime}<n$.

Let $\lambda_{I} \otimes u \in E_{1}^{s, t}(k)$ with $q=\left|\lambda_{I} \otimes u\right|=t-s \leq 2 k+1$. If $q<2 k$ then, by $1,2(\mathrm{i}), \lambda_{I} \otimes u$ lies in $E_{1}^{s, t}(k-1)$. Since $q \leq 2(k-1)+1$, by inductive hypothesis and the fact that $\beta_{*}$ commutes with all $d_{r}$, we see $d_{r}\left(\lambda_{I} \otimes u\right)=0$ for $r \leq n+1$. Thus we may assume $q=2 k$ or $2 k+1$.

By assumption, there exist a spectrum $W$ that satisfies " $C_{n-1}$ ", a map $f: Y$ $\rightarrow W$ monomorphic in homology, and a class $\nu \in H_{2|u|}(W)$ with $\nu S q^{|u|}=$ $f_{*}(u)$. Let $\left\{\bar{E}_{r}^{*, *}(m)\right\}$ be the Adams spectral sequence for $\pi_{*}^{s}(B(m) \wedge W)$. 
Now both $Y$ and $W$ satisfy " $C_{n-1}$ ". So, by inductive hypothesis, $E_{1}^{s, t}(k)=$ $E_{n+1}^{s, t}(k), \bar{E}_{1}^{s, t}(k)=\bar{E}_{n+1}^{s, t}(k)$ for $t-s \leq 2 k, \bar{E}_{1}^{s, t}(q)=\bar{E}_{n+1}^{s, t}(q) \quad\left(q=\left|\lambda_{I} \otimes u\right|=\right.$ $2 k$ or $2 k+1$ ) for $t-s \leq 2 q$ and $E_{n+1}^{s, t}(k)$ (resp. $\bar{E}_{n+1}^{s, t}(k)$ ) is a quotient $\mathbb{Z}_{2}$ module of $E_{1}^{s, t}(k)$ (resp. $\left.\bar{E}_{1}^{s, t}(k)\right)$ if $t-s=2 k+1$. Thus the induced map $f_{*}: E_{n+1}^{s, t}(k) \rightarrow \bar{E}_{n+1}^{s, t}(k)$ is also monomorphic for $t-s \leq 2 k$. Let $\pi: E_{1}^{s, t}(k) \rightarrow$ $E_{n+1}^{s, t}(k)$ (resp. $\left.\bar{\pi}: \bar{E}_{1}^{s, t}(k) \rightarrow \bar{E}_{n+1}^{s, t}(k)\right)$ be the quotient map if $t-s=2 k+1$ and the identity map if $t-s \leq 2 k$. We have

$$
\begin{aligned}
\left(f_{*} \circ d_{n+1} \circ \pi\right)\left(\lambda_{I} \otimes u\right) & =\left(\bar{d}_{n+1} \circ \bar{\pi}\right)\left(\lambda_{I} \otimes f_{*}(u)\right) \\
& =\left(\bar{d}_{n+1} \circ \bar{\pi}\right)\left(\lambda_{I} \otimes \nu S q^{|u|}\right) \\
& =\left(\bar{d}_{n+1} \circ \bar{\pi} \circ \alpha_{*}\right)\left(\lambda_{2 I} \otimes \nu\right) \quad(\text { by } 2(\mathrm{ii})) \\
& =\left(\alpha_{*} \circ \bar{d}_{n+1} \circ \bar{\pi}\right)\left(\lambda_{2 I} \otimes \nu\right) \\
& =0 .
\end{aligned}
$$

Here the last step follows by 2(i) since

$$
\bar{d}_{n+1}\left(\bar{\pi}\left(\lambda_{2 I} \otimes \nu\right)\right) \in \bar{E}_{n+1}^{s+n+1, s+n+2 q}(q)=\bar{E}_{1}^{s+n+1, s+n+2 q}(q)
$$

and

$$
\alpha_{*}\left(\bar{d}_{n+1}\left(\bar{\pi}\left(\lambda_{2 I} \otimes \nu\right)\right)\right) \in \bar{E}_{n+1}^{s+n+1, s+q+n}(k)=\bar{E}_{1}^{s+n+1, s+q+n}(k) .
$$

Since $d_{n+1}\left(\pi\left(\lambda_{I} \otimes u\right)\right)$ lies in $\bar{E}_{n+1}^{s, t}(k)$ with $t-s=q-1 \leq 2 k$ and since $f_{*}: E_{n+1}^{s, t}(k) \rightarrow \bar{E}_{n+1}^{s, t}(k)$ is monomorphic for $t-s \leq 2 k$ we see that

$$
d_{n+1}\left(\pi\left(\lambda_{I} \otimes u\right)\right)=0 \text {. }
$$

Thus $d_{n+1}\left(E_{n+1}^{s, s+q}\right)=0$ since $\pi$ is onto. This completes the inductive proof of Proposition 2.7 .

\section{REFERENCES}

1. J. F. Adams, On the non-existence of elements of Hopf invariant one, Ann. of Math. 72 (1960), 20-104.

2. Operations of the nth kind in $K$-theory and what we don't know about $\mathbb{R} p^{\infty}$, London Math. Soc. Lecture Note Ser., no. 11, Cambridge University, 1974, pp. 1-11.

3. M. F. Atiyah, Thom complexes, Proc. London. Math. Soc. 11 (1961), 291-310.

4. M. G. Barratt, J.D.S. Jones, and M. E. Mahowald, The Kervaire invariant problem, Contemp. Math., Amer. Math. Soc., vol. 19, 1983, pp. 9-22.

5. A. K. Bousfield, E. B. Curtis, D. M. Kan, D. G. Quillen, D. L. Rector, and J. W. Shlessinger, The mod-p lower central series and the Adams spectral sequence. II, Topology 9 (1970), 309-316.

6. E. H. Brown and R. L. Cohen, The Adams spectral sequence of $\Omega^{2} S^{3}$ and Brown-Gitler spectra, algebraic topology and algebraic $k$-theory (W. Browder, ed.), Ann. Math. Studies, no. 113, 1987, pp. 101-125.

7. E. H. Brown and S. Gitler, A spectrum whose cohomology is a certain cyclic module over the Steenrod algebra, Topology 12 (1973), 283-295.

8. E. H. Brown and F. P. Peterson, On the stable decomposition of $\Omega^{2} S^{r+2}$, Trans. Amer. Math. Soc. 243 (1978), 287-298. 
9. E. H. Brown and F. P. Peterson, The Brown-Gitler spectrum, $\Omega^{2} S^{3}$ and $\eta_{j} \in \pi_{2^{j}}(S)$, Trudy Mat. Inst. Steklov. 154 (1983), 38-43.

10. F. R. Cohen, M. E. Mahowald, and R. J. Milgram, The stable decomposition of the double loop space of a spheres, Proc. Sympos. Math., vol. 32, Amer. Math. Soc., Providence, RI, 1978, pp. 225-228.

11. D. M. Davis, The antiautomorphism of the Steenrod algebra, Proc. Amer. Math. Soc. 44 (1974), 235-236.

12. D. M. Davis and M. E. Mahowald, Classification of stunted real projective space, Pacific J. Math. 125 (1986), 335-345.

13. S. O. Kochman, A computer assisted approach to the calculation of stable stems (to appear).

14. M. E. Mahowald, A new infinite family in ${ }_{2} \pi_{*}^{S}$, Topology 16 (1977), 249-256.

15. M. E. Mahowald and M. C. Tangora, Some differentials in the Adams spectral sequence, Topology 6 (1967), 349-369.

16. R. J. Milgram and P. Zvengrowski, Even Whitehead squares are not projective, Canad. J. Math. 29 (1977), 957-962.

17. R. J. Milgram, J. Strutt, and P. Zvengrowsksi, Projective stable stems of spheres, Bol. Soc. Mat. Mexicana (2) 22 (1977), 48-57.

18. D. Randall, Projectivity of the Whitehead square, Proc. Amer. Math. Soc. 40 (1973), 609611.

19. V. P. Snaith, A stable decomposition of $\Omega^{n} S^{n} X$, J. London Math. Soc. 2 (1974), 577-583.

20. H. Toda, Composition methods in the homotopy groups of spheres, Ann. of Math. Stud., vol. 49, Princeton Univ. Press., Princeton, NJ, 1962.

21. J. H. C. Whitehead, On the groups $\pi_{*}\left(v_{n, m}\right)$ and sphere bundles, Proc. London Math. Soc. 48 (1944), 243-291.

Department of Mathematics, National Tsing Hua University, Hsinchu, Taiwan 30043 RePUblic OF CHINA 